\title{
Experience of Surgical Treatment in a Granular Cell Tumor in the Ascending Colon: A Case Report
}

\author{
In-Kyeong Kim ${ }^{1}$, Young-Tae Ju${ }^{1}$, Han-Gil Kim${ }^{1}$, Jin-Kwon Lee ${ }^{2}$, Dong-Chul Kim³ , Jae-Myung Kim ${ }^{1}$ \\ Jin Kyu Cho ${ }^{1}$, Ji-Ho Park ${ }^{1}$, Ju-Yeon Kim ${ }^{1}$, Chi-Young Jeong ${ }^{1}$, Soon-Chan Hong ${ }^{1}$, Seung-Jin Kwag ${ }^{1}$ \\ Departments of ${ }^{1}$ Surgery and ${ }^{3}$ Pathology, Gyeongsang National University Hospital, Gyeongsang National University College of Medicine, \\ Jinju; ${ }^{2}$ Department of Surgery, Gyeongsang National University Changwon Hospital, Gyeongsang National University College of Medicine, \\ Changwon, Korea
}

We report a case about successful surgical treatment of a granular cell tumor in the ascending colon. A 36-year-old man underwent screening colonoscopy. An endoscopic examination revealed a 10-mm yellowish and hemispheric mass in the ascending colon, and lower endoscopic ultrasonography revealed a hypoechoic-to-isoechoic mass invaded the submucosal layer. The mass was suspected to be a colonic carcinoid tumor. Based on the preoperative evaluation, endoscopic complete resection was considered difficult. Therefore, the lesion was removed via laparoscopic right hemicolectomy. Histological examination revealed that the tumor consisted of nests of polygonal cells with abundant granular eosinophilic cytoplasm. Immunohistochemical staining revealed diffuse positivity for S100 and CD68. Therefore, the tumor was diagnosed as a granular cell tumor. We suggest that surgical resection should be considered if it is located in the thin-walled ascending colon prone to perforation, difficult to rule out malignant tumor due to submucosal invasion, or to remove endoscopically.

Keywords: Granular cell tumor; Ascending colon; Operative surgical procedures

\section{INTRODUCTION}

Granular cell tumor (GCT) is a rare soft tissue neoplasm that arises from Schwann cells. Although a GCT can occur anywhere in the body, it is frequently observed in the oral cavity and subcutaneous tissues [1], and a common site in the gastrointestinal tract is the esophagus, followed by the duodenum, anus, and stomach [2]. Most colorectal GCTs develop as a solitary lesion in the proximal colon, cecum, appendix, and rectum [3], and these lesions are typically identified during colorectal examinations for other un-

Received: Sep 28, 2020 - Revised: May 6, 2021 - Accepted: May 8, 2021 Correspondence to: Seung-Jin Kwag, M.D.

Department of Surgery, Gyeongsang National University Hospital,

Gyeongsang National University College of Medicine, 79 Gangnam-ro, Jinju 52727, Korea

Tel: +82-55-750-8615, Fax: +82-55-757-5442

E-mail:drksj77@naver.com

ORCID: https://orcid.org/0000-0002-9267-9158

(C) 2021 The Korean Society of Coloproctology

This is an open-access article distributed under the terms of the Creative Commons Attribution NonCommercial License (https://creativecommons.org/licenses/by-nc/4.0) which permits unrestricted non-

commercial use, distribution, and reproduction in any medium, provided the original work is properly cited. derlying diseases or nonspecific symptoms. Although GCTs are usually benign, malignant tumors as reported in previous studies are likely to account for $1 \%$ to $2 \%$ of all GCTs [4]. Most cases of GCTs are treated via endoscopic submucosal dissection (ESD); however, ESD can be difficult to perform when GCT is located in the right-side colon, which is vulnerable to perforation or at risk of irregular submucosal invasion with the possibility of malignancy. We report the case of a man who was diagnosed with a GCT in the ascending colon and was successfully treated via laparoscopic right hemicolectomy.

\section{CASE REPORT}

This report was approved by the Institutional Review Board of Gyeongsang National University Hospital (No. 2020-06-029-001) and the patient provided informed consent for the publication of the report and accompanying images.

A 36-year-old Korean man visited Gyeongsang National University Hospital as an outpatient-based on abnormal results from a colonoscopy. He had a history of tuberculosis-related lung destruction with pneumothorax and 8-year smoking history. There 
was no other family or medical history. A physical examination revealed no symptoms related to the gastrointestinal tract and laboratory tests revealed normal findings for alpha-fetoprotein, carcinoembryonic antigen, and carbohydrate antigen 19-9. However, colonoscopy revealed an approximately $10-\mathrm{mm}$ yellowish protruding mass in the proximal ascending colon. The lesion was covered with intact mucosa and there was no rolling sign or cushion sign (Fig. 1). Lower endoscopic ultrasonography (EUS) also revealed an approximately $10-\mathrm{mm}$ hypoechoic-to-isoechoic mass mainly in the mucosal layer but also with irregular invasion of the submucosal layer. The muscularis propria appeared to be intact

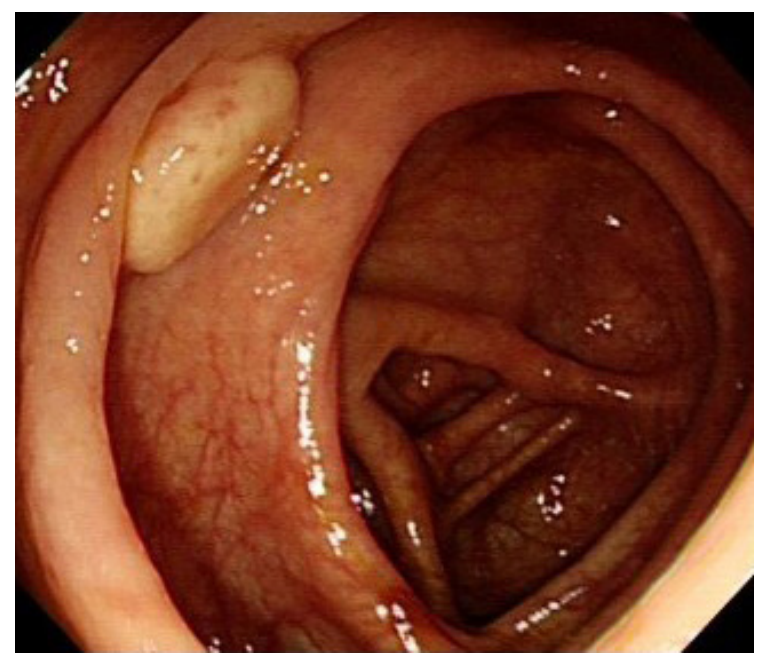

Fig. 1. Colonoscopy findings. A hemispheric yellowish protruding submucosal tumor with intact mucosa (approximate diameter, 10 $\mathrm{mm}$ ) was observed in the ascending colon.

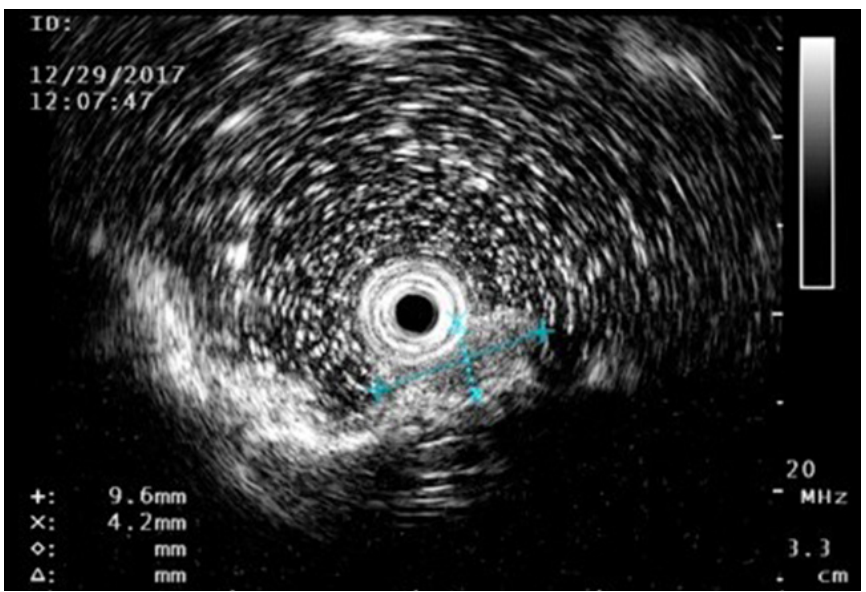

Fig. 2. Lower endoscopic ultrasonography revealed a hyperechoicto-isoechoic homogeneous tumor mainly in the muscularis mucosa, but with irregular invasion of the submucosa.
(Fig. 2). Therefore, the suspected diagnosis based on the EUS findings was a colonic carcinoid tumor. Abdominal computed tomography (CT) failed to identify the lesion and no metastatic lymph nodes were observed.

Findings from an endoscopic biopsy specimen were consistent with a GCT, although the possibility of a malignant tumor could not be completely excluded based on the endoscopic evaluation. Complete endoscopic resection was also considered difficult and surgical treatment was selected after a multidisciplinary team meeting. Laparoscopic right hemicolectomy with regional lymph node dissection was performed and negative proximal and distal margins were confirmed. The surgical findings revealed no evidence of lymph node enlargement or intraperitoneal metastasis. Gross examination of the specimen revealed a round solid hard mass (approximately $1.2 \times 1 \mathrm{~cm}$ ) that was adjacent to the ileocecal valve and on the mesentery aspect, with no enlargement of the lymph nodes (Fig. 3).

Histological examination revealed an elevated solid pale, yellowcolored tumor, with no metastasis in 14 regional lymph nodes. The tumor was confined to the mucosal layer and microscopic examination revealed a relatively well-circumscribed border located in the submucosa. The tumor consisted of nests of polygonal cells separated by thin collagenous fibers, with small roundto-oval nuclei, no pleomorphism, and abundant and slightly bright granular eosinophilic cytoplasm. There was no necrosis, hemorrhage, or mitosis. Immunohistochemical staining revealed that the tumor cells were diffusely positive for S100 and CD68. Therefore, the final diagnosis was GCT (Fig. 4). The patient did not experience any postoperative complications. Abdominal CT scans were performed 1 year after discharge and there was no evidence of local recurrence.

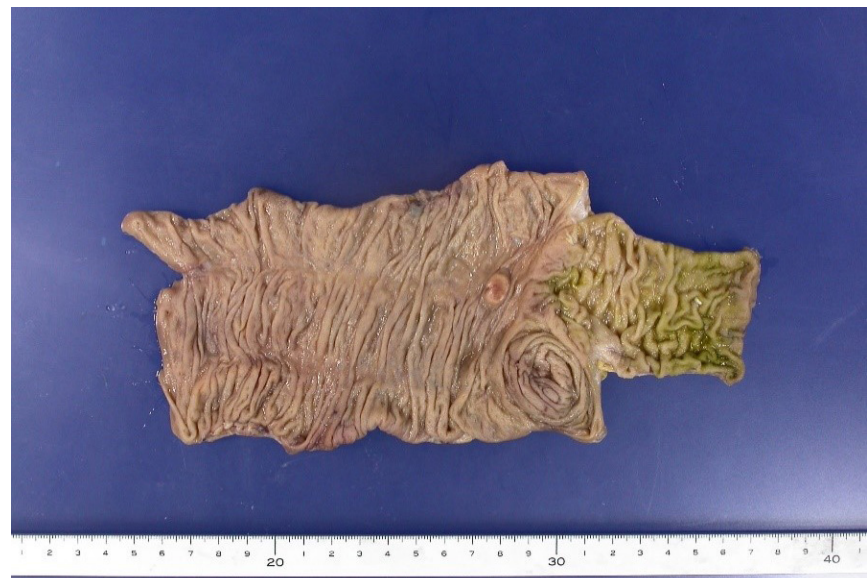

Fig. 3. Surgical findings. The specimen from the right colon resection consisted of the distal ileum (length, $7.5 \mathrm{~cm}$ ), cecum, and ascending colon (length, $14 \mathrm{~cm}$ ). The elevated solid pale, yellow-colored tumor was $1.2 \times 1 \mathrm{~cm}$ and located in the ascending colon near the ileocecal valve. 

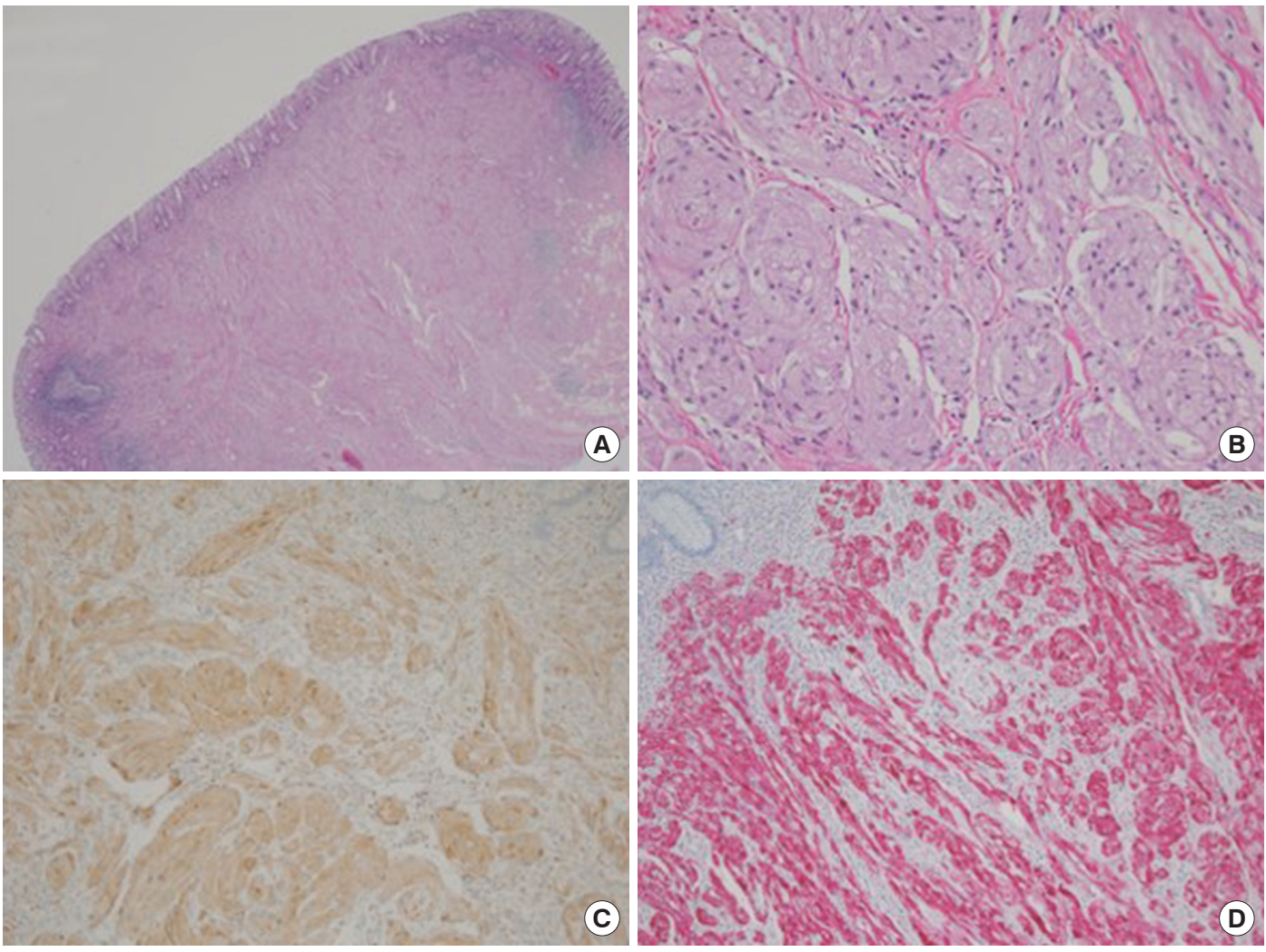

Fig. 4. Microscopic findings. (A) The resected tumor was located in the submucosa, was covered with normal mucosa, and had a relatively well-circumscribed border $(\mathrm{H} \& \mathrm{E}, \times 1.25)$. (B) The tumor consisted of nests of polygonal cells, which had small round-to-oval nuclei with no pleomorphism, as well as cytoplasm that was abundant, slightly granular, and brightly eosinophilic (H\&E, $\times 200)$. (C, D) Immunohistochemical staining revealed that the tumor cells were diffusely positive for CD68 (C) and S100 (D), but negative for normal epithelial cell markers.

\section{DISCUSSION}

A GCT is a rare and usually benign lesion that was first described by Abrikossoff in 1926 [5]. Although GCTs originate from Schwann cells and can develop anywhere in the body, GCTs are uncommon in the gastrointestinal tract, especially in the colon and rectum [1]. Because colonic GCTs tend to be asymptomatic, they are usually identified during colonoscopy screening or examinations for other reasons. The endoscopic findings for colonic GCTs are typically a yellow-white sessile small mass that is covered by apparently normal mucosa [6]. A recent report has indicated that EUS is essential for evaluating gastrointestinal submucosal tumors and determining the depth of their invasion [7], and GCTs generally appear as hypoechoic-to-isoechoic homogeneous lesions in the submucosal layer. However, yellowish hemispheric lesions in the mucosal or submucosal layer require a differential diagnosis that considers lipomas, cysts, metastatic lesions, and neurofibromas $[6,8]$. Furthermore, some reports have indicated that GCT needs to be differentiated from a carcinoid tumor [8] or carcinoma [9].

Yang et al. [10] reported a case in which GCT and rectal cancer were not distinguished. In the preoperative evaluation from abdominal CT, a 2-cm-sized rectal submucosal mass with mesorectal fat infiltration was diagnosed with stage $\mathrm{T} 3$ of rectal cancer. However, the final diagnosis was confirmed GCT by pathologic review. Therefore, the gross endoscopic appearance is not a definitive diagnosis and pathological findings are needed to confirm the diagnosis of colonic GCT. Nevertheless, Hwang et al. [7] have reported that the accuracy of EUS biopsy findings was limited for GCT (only 48\% accuracy in 23 cases), as it was difficult to determine the precise invasion extent of the hypoechoic lesion.

The final diagnosis of GCT is based on the pathological findings from the resected specimen. The tumor is characteristically comprised of plump neoplastic cells with abundant granular eosinophilic cytoplasm, which contains acidophilic periodic acid-Schiffpositive diastase-resistant granules and small uniform nuclei, but no mitotic figures. Immunohistochemical staining will reveal positivity for S100 protein or neuron-specific enolase, which are neural markers that suggest the tumor originates from Schwann cells $[6,11]$.

Complete excision is the standard treatment for gastrointestinal GCTs that are $>2 \mathrm{~cm}$ and infiltrating the muscularis propria, es- 
pecially if the lesion is suspected to be malignant. Endoscopic resection may be successful if the lesion is benign, has a diameter of $<2 \mathrm{~cm}$, and has not invaded the muscularis propria [12]. Endo et al. [6] have reported that endoscopic resection was performed for $78.8 \%$ of colonic GCTs in their study, whereas Nakachi et al. [9] recommended endoscopic resection and regular endoscopic follow-up for lesions that were asymptomatic, $<2 \mathrm{~cm}$ in size, and had typical findings. Nevertheless, ESD is not indicated for lesions with substantial submucosal invasion, as en bloc resection can lead to bowel perforation [13]. Moreover, lesions in the colon are difficult to treat without complications, and ESD is difficult to perform in the right colon, given the difficulty of navigating the colon and reaching the lesion. Moreover, the wall of the right colon is thin and distensible, which increases the risk of perforation. Therefore, ESD in the right colon should only be attempted by a skilled physician in cases where it is absolutely necessary [14].

Although GCTs are generally considered benign, a few cases have involved malignant GCTs, with malignant potential estimated in $1 \%$ to $2 \%$ of GCT cases $[4,15]$. These tumors have been classified as histologically and clinically malignant or histologically benign and clinically malignant. Malignancy is histologically diagnosed based on the presence of $\geq 3$ of the following features: spindle cells, the presence of vesicular nuclei with large nucleoli, an increased mitotic rate, a high nuclear-to-cytoplasmic ratio, pleomorphism, and necrosis. Malignancy may also be identified based on clinical findings, which include metastasis, large size, rapid growth, and invasion into adjacent tissues, even if the histological findings do not clearly indicate malignancy [13]. In particular, malignancy is related to GCT size, with $>60 \%$ of metastatic GCTs being $>4 \mathrm{~cm}[6]$. Therefore, surgical resection may be preferred to ESD in cases that involve a GCT that has a size of $>4$ $\mathrm{cm}$, a lesion located in the right colon, enlarged lymph nodes, lymphovascular metastasis, or malignant potential based on the preoperative examination.

In the present case, the colonic GCT had a size of $<2 \mathrm{~cm}$, although the preoperative biopsy provided insufficient histological evidence and a carcinoid tumor could not be ruled out based on the EUS results. Thus, surgical resection was selected rather than ESD because of the irregular invasion of the submucosal layer and the high risk of perforation when performing ESD in the right colon. Furthermore, histological findings that suggested a benign lesion could not completely exclude the possibility of a malignant lesion. Therefore, after careful consultation with the patient, surgical resection was performed and the patient did not experience any complications. Therefore, gastroenterologists, surgeons, and pathologists should consider appropriate treatment based on the possibility of malignancy and a differential diagnosis that considers GCT and other lesions.

There are currently no guidelines for colorectal GCTs, but in cases which the possibility of malignancy or the risk of bowel perforation cannot be completely ruled out, radical resection could be considered. Malignant GCT has shown poor prognosis in pre- vious studies that reported local recurrence rate of $30 \%$ to $40 \%$ and metastasis rate of $50 \%$ to $60 \%$ in the reviewed cases [15].

In conclusion, we encountered a patient with a GCT in the ascending colon, showing that colonic GCTs can be found incidentally during colonoscopy. EUS is generally useful in differential diagnosis of gastrointestinal submucosal tumors. However, the findings from EUS may not exclude the possibility of malignancy such as irregular invasion of submucosal layer, or the differentiation of carcinoid tumor, even for small GCTs. Moreover, the location of the tumor is at risk for endoscopic resection such as perforation. Therefore, surgical excision with wide margins may be an appropriate treatment for an uncertain tumor invading the submucosal layer of the ascending colon.

\section{CONFLICT OF INTEREST}

No potential conflict of interest relevant to this article was reported.

\section{ACKNOWLEDGMENTS}

This study was supported by grants of the Basic Science Research Program through the National Resarch Foundation of Korea (NRF) funded by the Ministry of Science, ICT and Future Planning. Republic of Korea. ( No.NRF-2018R1D1A1B07045469).

\section{REFERENCES}

1. Yasuda I, Tomita E, Nagura K, Nishigaki Y, Yamada O, Kachi H. Endoscopic removal of granular cell tumors. Gastrointest Endosc 1995;41:163-7.

2. Lack EE, Worsham GF, Callihan MD, Crawford BE, Klappenbach S, Rowden G, et al. Granular cell tumor: a clinicopathologic study of 110 patients. J Surg Oncol 1980;13:301-16.

3. Sohn DK, Choi HS, Chang YS, Huh JM, Kim DH, Kim DY, et al. Granular cell tumor of colon: report of a case and review of literature. World J Gastroenterol 2004;10:2452-4.

4. Fanburg-Smith JC, Meis-Kindblom JM, Fante R, Kindblom LG. Malignant granular cell tumor of soft tissue: diagnostic criteria and clinicopathologic correlation. Am J Surg Pathol 1998;22:77994.

5. Abrikossoff A. Über Myome - ausgehend von der quergestreiften willkürlichen Muskulatur. Virchows Arch Pathol Anat Physiol Klin Med 1926;260:215-33.

6. Endo S, Hirasaki S, Doi T, Endo H, Nishina T, Moriwaki T, et al. Granular cell tumor occurring in the sigmoid colon treated by endoscopic mucosal resection using a transparent cap (EMR-C). J Gastroenterol 2003;38:385-9.

7. Hwang JH, Saunders MD, Rulyak SJ, Shaw S, Nietsch H, Kimmey MB. A prospective study comparing endoscopy and EUS in the evaluation of GI subepithelial masses. Gastrointest Endosc 2005; 62:202-8. 
8. Shimoyama M, Sakai Y, Takaku H, Takii Y, Okamoto H, Suda T, et al. A case of multiple granular cell tumors in the cecum. Gastroenterol Endosc 1999;41:1330-5.

9. Nakachi A, Miyazato H, Oshiro T, Shimoji H, Shiraishi M, Muto Y. Granular cell tumor of the rectum: a case report and review of the literature. J Gastroenterol 2000;35:631-4.

10. Yang SY, Min BS, Kim WR. A granular cell tumor of the rectum: a case report and review of the literature. Ann Coloproctol 2017;33: 245-8.

11. Cavaliere A, Sidoni A, Ferri I, Falini B. Granular cell tumor: an immunohistochemical study. Tumori 1994;80:224-8.

12. Choi SM, Hong SG, Kang SM, Chae BG, Kim SJ, Park PK, et al. A case of malignant granular cell tumor in the sigmoid colon. Clin Endosc 2014;47:197-200.

13. Tanaka S, Oka S, Chayama K. Colorectal endoscopic submucosal dissection: present status and future perspective, including its differentiation from endoscopic mucosal resection. J Gastroenterol 2008;43:641-51.

14. Kim ER, Chang DK. Management of complications of colorectal submucosal dissection. Clin Endosc 2019;52:114-9.

15. Mahoney A, Garg A, Wolpowitz D, Mahalingam M. Atypical granular cell tumor-apropos of a case with indeterminate malignant potential. Am J Dermatopathol 2010;32:370-3. 